\title{
THE QUANTUM SPHERES AND THEIR EMBEDDING INTO QUANTUM MINKOWSKI SPACE-TIME
}

\author{
M. LAGRAA
}

Received 3 November 2001 and in revised form 12 April 2002

We recast the Podleś spheres in the noncommutative physics context by showing that they can be regarded as slices along the time coordinate of the different regions of the quantum Minkowski space-time. The investigation of the transformations of the quantum sphere states under the left coaction of the $\mathrm{SO}_{q}(3)$ group leads to a decomposition of the transformed Hilbert space states in terms of orthogonal subspaces exhibiting the periodicity of the quantum sphere states.

\section{Introduction}

A great variety of works based on the quantum spheres have been developed since the appearance of Podleś spheres [11] and their symmetries [12]. Most part of these studies have been done either in the quantum bundle formalism where the quantum spheres provide concrete examples to test the different structures of this formalism $[1,5,6]$ or, more recently, in quantum field theories on quantum spheres which should respect the $\mathrm{SU}_{q}(2)$ quantum symmetries (see, e.g., $[2,3,4,10]$ and the references therein).

On the other hand, the evolution of a free particle in the quantum Minkowski space-time has been analysed in [8] and the transformations of its quantum velocity under the Lorentz subgroup of boost transformations in [7].

In Section 2 of this paper, we pursue these studies by recasting the quantum spheres in the noncommutative special relativity where we show that we can regard them as quantum manifolds embedded into the quantum Minkowski space-time. This embedding preserves the reality 
structure and the commutations rules of the quantum Minkowski spacetime coordinates. In particular, we show that in the time-like region of the quantum Minkowski space-time the Hilbert space $\mathscr{L}^{(L)}$ of states describing the noncommutative relativistic evolution of a free particle having a quantum velocity of length $|v|_{q}^{2}=\left(1+Q^{2} c(L)\right), c(L)=-1 /\left(q^{(L+1)}+\right.$ $\left.q^{-(L+1)}\right)^{2}$ with $L \geq 1$ is an integer, $q$ is the deformation parameter and $Q=q+q^{-1}$ are precisely, for fixed time, the space of irreducible representations of the Podleś quantum spheres $S_{q c}^{2}$ with $c=c(L)$. We also show in this section that the Hilbert space of representations of the space-like region of the quantum Minkowski space-time corresponds, for particular fixed time, to the Hilbert space of representations of the quantum spheres $S_{q c}^{2}$, where $c \in[0, \infty]$ or $S_{q \infty}^{2}$.

In Section 3, we show that the state transformations under the coaction of the $\mathrm{SO}_{q}(3)$ group exhibits the periodicity of the quantum sphere states through a decomposition of the transformed Hilbert space in terms of orthogonal subspaces, each describes the same quantum sphere.

\section{The quantum spheres}

Before embedding the different quantum spheres into the quantum Minkowski space-time, we recall briefly some properties of the noncommutative special relativity presented in [8]. First, it was shown in [9] that the generators $\Lambda_{N}{ }^{M}(N, M=0,1,2,3)$ of quantum Lorentz group may be written in terms of those of quantum $\operatorname{SL}(2, C)$ group as

$$
\Lambda_{N}{ }^{M}=\frac{1}{Q} \varepsilon_{\dot{\gamma} \dot{\delta}} \bar{\sigma}_{N} \dot{\delta} \alpha M_{\alpha}{ }^{\sigma} \sigma^{M}{ }_{\sigma \dot{\rho}} M_{\dot{\beta}} \dot{\rho} \varepsilon^{\dot{\gamma} \dot{\beta}},
$$

where $M_{\alpha} \beta(\alpha, \beta=1,2)$ and $M_{\dot{\alpha}} \dot{\beta}=\left(M_{\alpha}^{\beta}\right)^{\star}$ are the generators of the quantum $\mathrm{SL}(2, C)$ group subject to the unimodularity conditions

$$
\begin{gathered}
\varepsilon_{\alpha \beta} M_{\gamma}{ }^{\alpha} M_{\delta}{ }^{\beta}=\varepsilon_{\gamma \delta}, \quad \varepsilon^{\gamma \delta} M_{\gamma}{ }^{\alpha} M_{\delta}{ }^{\beta}=\varepsilon^{\alpha \beta}, \\
\varepsilon_{\dot{\alpha} \dot{\beta}} M_{\dot{\gamma}}{ }^{\dot{\alpha}} M_{\dot{\delta}}^{\dot{\beta}}=\varepsilon_{\dot{\gamma} \dot{\delta}}, \quad \varepsilon^{\dot{\gamma}} \dot{\delta} M_{\dot{\gamma}}{ }^{\dot{\alpha}} M_{\dot{\delta}} \dot{\dot{\beta}}=\varepsilon^{\dot{\alpha} \dot{\beta}}, \quad Q=q+q^{-1}
\end{gathered}
$$

and the spinor metrics are taken to be $\varepsilon_{\alpha \beta}=-\varepsilon^{\dot{\beta} \dot{\alpha}}=\left(\begin{array}{cc}0-q^{-1 / 2} \\ q^{1 / 2} & 0\end{array}\right)$, where $q \in$ ]0,1[ is a real deformation parameter. $\sigma_{\alpha \dot{\beta}}^{N}$ are a set of four independent matrices composed by the Pauli matrices $\sigma_{\alpha \dot{\beta}}^{n}(n=1,2,3)$ and the identity matrix $\sigma_{\alpha \dot{\beta}}^{0}$ and

$$
\bar{\sigma}_{ \pm}^{I \dot{\alpha} \beta}=\varepsilon^{\dot{\alpha} \dot{\lambda}} R^{-\sigma \dot{\rho}} \dot{i \nu}_{\nu} \varepsilon^{\nu \beta} \sigma_{\sigma \dot{\rho}}^{I}=q^{1 / 2} \varepsilon^{\dot{\lambda} \dot{\rho}} R^{-\sigma \lambda}{ }_{\alpha \nu} \varepsilon^{\nu \beta} \sigma_{\sigma \dot{\rho}}^{I} .
$$


The $R$-matrices are given by

$$
R_{\alpha \gamma}^{ \pm \delta \beta}=\delta_{\alpha}^{\delta} \delta_{\gamma}^{\beta}+q^{ \pm 1} \varepsilon^{\delta \beta} \varepsilon_{\alpha \gamma}, \quad R^{\dot{\alpha} \dot{\gamma}}{ }_{ \pm \dot{\delta} \dot{\beta}}=\delta_{\dot{\alpha}}^{\dot{\delta}} \delta_{\dot{\gamma}}^{\dot{\beta}}+q^{ \pm 1} \varepsilon^{\dot{\delta} \dot{\beta}} \varepsilon_{\dot{\alpha} \dot{\gamma}}
$$

satisfying $R_{\alpha \gamma}^{ \pm \delta \beta} R_{\delta \beta}^{\mp \rho \sigma}=\delta_{\alpha}^{\rho} \delta_{\gamma}^{\sigma}$ and $R^{ \pm \dot{\delta} \dot{\beta}} \dot{\alpha} \dot{\gamma} R^{\mp \dot{\rho} \dot{\sigma}} \dot{\delta} \dot{\beta}=\delta_{\dot{\alpha}}^{\dot{\rho}} \delta_{\dot{\gamma}}^{\dot{\sigma}}$. They induce the commutation rules

$$
\begin{aligned}
& M_{\alpha}{ }^{\rho} M_{\beta}{ }^{\sigma} R_{\rho \sigma}^{ \pm \gamma \delta}=R_{\alpha \beta}^{ \pm \rho \sigma} M_{\rho}^{\gamma} M_{\sigma}{ }^{\delta}, \\
& M_{\dot{\alpha}}{ }^{\dot{\rho}} M_{\dot{\beta}}^{\dot{\sigma}} R^{ \pm \dot{\gamma} \dot{\delta}} \dot{\rho} \dot{\sigma}=R_{\dot{\alpha} \dot{\beta}}^{ \pm \dot{\rho} \dot{\rho}} M_{\dot{\rho}}^{\dot{\gamma}} M_{\dot{\sigma}}^{\dot{\delta}} .
\end{aligned}
$$

The Lorentz group generators are real, $\left(\Lambda_{N}{ }^{M}\right)^{\star}=\Lambda_{N}{ }^{M}$, and generate a Hopf algebra $\mathcal{L}$ endowed with a coaction $\Delta$, a counit $\varepsilon$, and an antipode $S$ acting as $\Delta\left(\Lambda_{N}{ }^{M}\right)=\Lambda_{N}{ }^{K} \otimes \Lambda_{K}{ }^{M}, \varepsilon\left(\Lambda_{N}{ }^{M}\right)=\delta_{N}^{M}$, and $S\left(\Lambda_{N}{ }^{M}\right)=$ $G_{N K} \Lambda_{L}{ }^{K} G^{L M}$, respectively. $G^{N M}$ is an invertible and hermitian quantum metric given by

$$
G^{I J}=\left(\frac{1}{Q}\right) \varepsilon^{\alpha \nu} \sigma_{\alpha \dot{\beta}}^{I} \bar{\sigma}^{J \dot{\beta} \gamma} \varepsilon_{\gamma \nu}=\left(\frac{1}{Q}\right) \varepsilon_{\dot{\nu} \dot{\gamma}} \bar{\sigma}^{I \dot{\gamma} \alpha} \sigma_{\alpha \dot{\beta}}^{J} \varepsilon^{\dot{\nu} \dot{\beta}} .
$$

The form of the antipode of $\Lambda_{N}{ }^{M}$ implies the orthogonality conditions $G_{N M} \Lambda_{L}{ }^{N} \Lambda_{K}{ }^{M}=G_{L K}$ and $G^{L K} \Lambda_{L}{ }^{N} \Lambda_{K}{ }^{M}=G^{N M}$ of the generators of the quantum Lorentz group.

The quantum metric $G^{N M}$ can be considered as a metric of a quantum Minkowski space-time $\boldsymbol{M}_{4}$ equipped with real coordinates $X_{N},\left(X_{N}\right)^{\star}=$ $X_{N} \cdot X_{0}$ represents the time operator and $X_{i}(i=1,2,3)$ represent the space right invariant coordinates, $\Delta_{R}\left(X_{I}\right)=X_{I} \otimes I$, which transform under the left coaction as

$$
\Delta_{L}\left(X_{I}\right)=\Lambda_{I}^{K} \otimes X_{K}
$$

From the hermiticity of the Minkowskian metric and the orthogonality conditions, we can see that the four-vector length $G^{N M} X_{N} X_{M}=-\tau^{2}$ is real and invariant. It was also shown in [9] that $\tau^{2}$ is central; it commutes with the Minkowski space-time coordinates and the quantum Lorentz group generators. $\Lambda_{N}{ }^{M}$ and $X_{N}$ are subject to the commutation rules controlled by the $R_{P Q}^{N M}$ matrix as

$$
\begin{aligned}
\Lambda_{L}{ }^{P} \Lambda_{K}{ }^{Q} \mathcal{R}_{P Q}^{N M} & =R_{L K}^{P Q} \Lambda_{P}{ }^{N} \Lambda_{Q}{ }^{M}, \\
X_{N} X_{M} & =\mathcal{R}_{N M}^{P Q} X_{P} X_{Q},
\end{aligned}
$$


where the $R$-matrix of the Lorentz group is constructed out of those of $\operatorname{SL}(2, C)$ group and satisfies the relations $R_{K L}^{N M} G^{K L}=G^{N M}$ and $R_{K L}^{N M} G_{N M}$ $=G_{K L}$ which show the quantum symmetrization of the Minkowskian metric $G^{N M}$ and its inverse.

To make an explicit calculation of the different commutation rules of the generators of the quantum Lorentz group, we take the following choice of Pauli hermitian matrices

$$
\begin{array}{ll}
\sigma_{\alpha \dot{\beta}}^{0}=\left(\begin{array}{cc}
1 & 0 \\
0 & 1
\end{array}\right), & \sigma_{\alpha \dot{\beta}}^{1}=\left(\begin{array}{cc}
0 & 1 \\
1 & 0
\end{array}\right), \\
\sigma_{\alpha \dot{\beta}}^{2}=\left(\begin{array}{cc}
0 & -i \\
i & 0
\end{array}\right), & \sigma_{\alpha \dot{\beta}}^{3}=\left(\begin{array}{cc}
q & 0 \\
0 & -q^{-1}
\end{array}\right) .
\end{array}
$$

This choice leads us to a quantum metric form $G^{L K}$ exhibiting two independent blocks, one for the time index and the others for space components indices $(k=1,2,3)$ whose nonvanishing elements are $G^{00}=-q^{-3 / 2}$, $G^{11}=G^{22}=G^{33}=q^{1 / 2}, G^{12}=-G^{21}=-i q^{1 / 2}\left(\left(q-q^{-1}\right) / Q\right)$. The nonvanishing elements of its inverse are $G_{00}=-q^{3 / 2}, G_{11}=G_{22}=q^{-1 / 2}\left(Q^{2} / 4\right), G_{33}=$ $q^{-1 / 2}$, and $G_{12}=-G_{21}=i q^{-1 / 2}\left(\left(q-q^{-1}\right) Q / 4\right)$. In the classical limit $q=1$, this metric reduces to the classical Minkowski metric with signature $(-,+,+,+)$. Explicitly, the length of the four-vector $X_{N}$ reads

$$
G^{N M} X_{N} X_{M}=-\tau^{2}=-q^{-3 / 2} X_{0}^{2}+q^{1 / 2}\left(\frac{q X_{z} X_{\bar{z}}+q^{-1} X_{\bar{z}} X_{z}}{Q}+X_{3}^{2}\right)
$$

where $X_{z}=X_{1}+i X_{2}$ and $X_{\bar{z}}=X_{1}-i X_{2}$. An explicit computation of (2.9) gives

$$
\begin{gathered}
{\left[X_{0}, X_{N}\right]=0} \\
X_{3} X_{z}-q^{2} X_{z} X_{3}=\left(q-q^{-1}\right) X_{0} X_{z} \\
X_{3} X_{\bar{z}}-q^{-2} X_{\bar{z}} X_{3}=-q^{-2}\left(q-q^{-1}\right) X_{0} X_{\bar{z}} \\
X_{z} X_{\bar{z}}-X_{\bar{z}} X_{z}=\left(q-q^{-1}\right) Q\left(X_{3}^{2}+q^{-1} X_{0} X_{3}\right) .
\end{gathered}
$$

The Pauli matrices satisfy $\bar{\sigma}_{0}^{\dot{\alpha} \beta}=-\sigma_{\alpha \dot{\beta}}^{0}=-\delta_{\alpha}^{\beta}, \bar{\sigma}_{N \dot{\alpha} \alpha}=\bar{\sigma}_{N i 1}+\bar{\sigma}_{N \dot{2} 2}=-Q \delta_{N}^{0}$ and $\sigma^{N \alpha \dot{\alpha}}=Q \delta_{0}^{N}$ which make explicit the restriction of the quantum Lorentz group to the quantum subgroup of the three-dimensional space rotations by restricting the quantum $\operatorname{SL}(2, C)$ group generators to those of the $S U(2)$ group. In fact when we impose the unitarity conditions, $M_{\dot{\alpha}} \dot{\beta}=S\left(M_{\beta}{ }^{\alpha}\right)$, in (2.1) we get

$$
\Lambda_{N}{ }^{0}=\delta_{N^{\prime}}^{0} \quad \Lambda_{0}^{M}=\delta_{0}^{M},
$$


which lead us to the restriction of the Minkowski space-time transformations under the quantum Lorentz group to the orthogonal transformations group $\mathrm{SO}_{q}(3)$. This subgroup leaves invariant the three-dimensional quantum subspace $\boldsymbol{R}_{3} \subset \mathcal{M}_{4}$ equipped with the real coordinate system $X_{i}(i=1,2,3)$ and the Euclidean metric $G^{i j}$. More precisely as a consequence of $(2.14),(2.7)$ reduces to

$$
\bar{\Delta}_{L}\left(X_{0}\right)=\bar{\Lambda}_{0}^{0} \otimes X_{0}=I \otimes X_{0}, \quad \bar{\Delta}_{L}\left(X_{i}\right)=\bar{\Lambda}_{i}^{j} \otimes X_{j}
$$

where $\bar{\Delta}_{(L)}$ is the restriction of (2.7) to the three-dimensional quantum subspace $R_{3}$ of $\mathcal{M}_{4} \cdot \bar{\Lambda}_{i}^{j}=(1 / Q) \bar{\sigma}_{i \dot{\gamma}}{ }^{\alpha} M_{\alpha}{ }^{\sigma} \sigma^{j}{ }_{\sigma \dot{\rho}} S\left(M_{\rho}{ }^{\beta}\right) \varepsilon^{\dot{\gamma} \dot{\beta}}$ generate an $\mathrm{SO}_{q}$ (3) Hopf subalgebra $\mathcal{L}$ whose axiomatic structure is derived from those of $\mathcal{L}$ as $\Delta\left(\bar{\Lambda}_{i}{ }^{j}\right)=\bar{\Lambda}_{i}{ }^{k} \otimes \bar{\Lambda}_{k}{ }^{j}, \varepsilon\left(\bar{\Lambda}_{i}{ }^{j}\right)=\delta_{i}{ }^{j}$ and $S\left(\bar{\Lambda}_{i}{ }^{j}\right)=G_{i K} \bar{\Lambda}_{L}{ }^{K} G^{L j}=$ $G_{i k} \bar{\Lambda}_{l}{ }^{k} G^{l j}$, where $G^{i j}$ is the restriction of $G^{I J}$ satisfying $G^{i k} G_{k j}=\delta_{j}^{i}=$ $G_{j k} G^{k i}, G^{i j} R_{i j}^{k l}=G^{k l}$ and $G_{k l} R_{i j}^{k l}=G_{i j}$ which are the quantum symmetrization of the Euclidean metric $G^{i j}$ and its inverse. The form of the antipode $S\left(\bar{\Lambda}_{i}{ }^{j}\right)$ implies the orthogonality properties of the generators of the quantum subgroup $\mathrm{SO}_{q}(3)$ as $G^{i j} \bar{\Lambda}_{i}^{l} \bar{\Lambda}_{j}{ }^{k}=G^{l k}$ and $G_{l k} \bar{\Lambda}_{i}{ }^{l} \bar{\Lambda}_{i}{ }^{k}=G_{i j}$. The commutation rules of the coordinate $X_{i}$ of $\mathcal{R}_{3}$ satisfy the same commutation rules (2.13), where $X_{0}$ is taken to be a constant parameter recalling that it commutes with the spacial coordinates $X_{i}$.

Therefore, $\bar{\Lambda}_{i}^{j}=(1 / Q) \bar{\sigma}_{i \dot{\gamma}}{ }^{\alpha} M_{\alpha}{ }^{\sigma} \sigma_{\sigma \dot{\rho}}^{j} S\left(M_{\rho}{ }^{\beta}\right) \varepsilon^{\dot{\gamma} \dot{\beta}}$ establishes a correspondence between $\mathrm{SU}_{q}(2), M_{\alpha}^{\beta}=\left(\begin{array}{cc}\alpha & -q \gamma^{\star} \\ \gamma & \alpha^{\star}\end{array}\right)$ with the commutation relation

$$
\begin{gathered}
\alpha \alpha^{\star}+q^{2} \gamma \gamma^{\star}=1, \quad \alpha^{\star} \alpha+\gamma \gamma^{\star}=1, \quad \gamma \gamma^{\star}=\gamma^{\star} \gamma, \\
\alpha \gamma^{\star}=q \gamma^{\star} \alpha, \quad \alpha \gamma=q \gamma \alpha
\end{gathered}
$$

and $\mathrm{SO}_{q}(3)$ group. In the three-dimensional space $\mathcal{R}_{3}$ spanned by the basis $X_{z}, X_{\bar{z}}$, and $X_{3}$, where the $\mathrm{SO}_{q}(3)$ coacts, the generators $\bar{\Lambda}_{i}{ }^{j}=$ $(1 / Q) \bar{\sigma}_{i \dot{\gamma}}{ }^{\alpha} M_{\alpha}{ }^{\sigma} \sigma_{\sigma \dot{\rho}}^{j} S\left(M_{\rho}{ }^{\beta}\right) \varepsilon^{\dot{\gamma} \dot{\beta}}$ read

$$
\left(\bar{\Lambda}_{i}^{j}\right)=\left(\begin{array}{ccc}
-2 q \gamma \gamma & 2 \alpha^{\star} \alpha^{\star} & Q \gamma \alpha^{\star} \\
2 \alpha \alpha & -2 q \gamma^{\star} \gamma^{\star} & Q \alpha \gamma^{\star} \\
-2 \alpha \gamma & -2 \gamma^{\star} \alpha^{\star} & 1-q Q \gamma \gamma^{\star}
\end{array}\right) \in M_{3} \otimes C\left(\operatorname{SU}_{q}(2)\right)
$$

where the indices $i, j$ run over $z=1+i 2, \bar{z}=1-i 2$ and 3 .

In the case $\tau^{2}>0$, time-like region, it was shown in [8] that the evolution of a free particle in the Minkowski space-time is described by states belonging to the Hilbert space $\mathscr{\ell}^{(L)}$ whose basis is spanned by common 
eigenstate of $X_{0}$ and $X_{3}$

$$
X_{0}|t, L, n\rangle=t|t, L, n\rangle, \quad x_{3}|t, L, n\rangle=x_{3}^{(L, n)}|t, L, n\rangle,
$$

where $\tau^{2}=q^{-3 / 2}\left(t^{2} / \gamma^{2(L)}\right), x_{3}^{(L, n)}=q^{-1} t\left(q^{-(L-2 n)} / \gamma^{(L)}-1\right), \gamma^{(L)}=\left(q^{(L+1)}+\right.$ $\left.q^{-(L+1)}\right) / Q, L=0,1,2, \ldots, \infty$, and $n$ runs by integer steps over the range $0 \leq n \leq L . X_{z}$ and $X_{\bar{z}}$ act on the basis elements of $\mathscr{L}^{(L)}$ as

$$
\begin{aligned}
& X_{z}|t, L, n\rangle=\lambda \frac{q^{-1} t}{\gamma^{(L)}} q^{-(L-n)}\left(1-q^{2(n+1)}\right)^{1 / 2}\left(1-q^{2(L-n)}\right)^{1 / 2}|t, L, n+1\rangle, \\
& X_{\bar{z}}|t, L, n\rangle=\bar{\lambda} \frac{q^{-1} t}{\gamma^{(L)}} q^{-(L-n+1)}\left(1-q^{2 n}\right)^{1 / 2}\left(1-q^{2(L-n+1)}\right)^{1 / 2}|t, L, n-1\rangle,
\end{aligned}
$$

respectively, where $\lambda \bar{\lambda}=1$. In what follows, we will take $\lambda=-1$. The length of velocity of the particle is given by $|\vec{v}|_{q}^{2}=q^{2}\left(\left(q V_{z} V_{\bar{z}}+q^{-1} V_{\bar{z}} V_{z}\right) /\right.$ $\left.Q+V_{3}^{2}\right)=1-1 / \gamma^{2(L)} \leq 1$.

The light-cone, $\tau^{2}=0$, corresponds to $L=\infty$ leading to $|\vec{v}|_{q}^{2}=1$ which is the velocity of the light. In this region, the evolution of the particle is described by states $|t, n\rangle(n=0,1, \ldots, \infty)$ satisfying

$$
\begin{aligned}
& X_{0}|t, n\rangle=t|t, n\rangle, \\
& X_{3}|t, n\rangle=q^{-1} t\left(q^{2 n+1} Q-1\right)|t, n\rangle, \\
& X_{z}|t, n\rangle=-q^{n} t Q\left(1-q^{2(n+1)}\right)^{1 / 2}|t, n+1\rangle, \\
& X_{\bar{z}}|t, n\rangle=-q^{(n-1)} t Q\left(1-q^{2 n}\right)^{1 / 2}|t, n-1\rangle .
\end{aligned}
$$

In what follows, we will take the length of the quantum three-vector as $|\vec{X}|_{q}^{2}=\left(q X_{z} X_{\bar{z}}+q^{-1} X_{\bar{z}} X_{z}\right) / Q+X_{3}^{2}$. The quantum group $\mathrm{SO}_{q}(3)$ acts on the spatial coordinates $X_{i}$ as (2.15) and lives invariant both $X_{0}$ and $\tau^{2}$, then $q^{-2} X_{0}^{2}-q^{-1 / 2} \tau^{2}=R^{2}=q^{-1 / 2} G^{i j} X_{i} X_{j}=\left(\left(q X_{z} X_{\bar{z}}+q^{-1} X_{\bar{z}} X_{z}\right) / Q+X_{3}^{2}\right)=$ $|\vec{X}|_{q}^{2}$. For fixed $t^{2}$, we have

$$
R^{2(L)}|L, n\rangle=q^{-2} t^{2}\left(1-\frac{1}{r^{2(L)}}\right)|L, n\rangle
$$

and the relations (2.18) and (2.20) for finite $L \geq 1$ and

$$
R^{2}|n\rangle=q^{-2} t^{2}|n\rangle
$$


and the relations (2.22) for $L=\infty$, where the orthonormal states $|L, n\rangle$ and $|n\rangle,\left\langle L, n^{\prime} \mid L, n\right\rangle=\delta_{n^{\prime}, n}, n^{\prime}, n=0,1, \ldots, L$, and $\left\langle n^{\prime} \mid n\right\rangle=\delta_{n^{\prime}, n}, n^{\prime}, n=$ $0,1, \ldots, \infty$, denote the states satisfying (2.18), (2.20), and (2.22), respectively. The unique state $|t, 0,0\rangle$ corresponding to $L=0$ describes a particle at rest at the origin of the spacial coordinate system, for $t=0$, then $\tau^{2}=0$ represents the origin of the four coordinate system of the quantum Minkowski space-time, $X_{N}|0,0,0\rangle=0|0,0,0\rangle$. Therefore, the $(L+1)$ dimensional Hilbert subspace $\mathscr{H}^{(L)}$ of states describing the evolution of a free particle of a given length of the velocity in the noncommutative Minkowski space-time can be identified, for fixed time, with the Hilbert space $\mathscr{\ell}_{S_{q}^{2}}^{(L)}$ of irreducible representations of the quantum spheres of radius $R^{(L)}=q^{-1} t\left(1-1 / \gamma^{2(L)}\right)^{1 / 2}$. This observation leads us to state the following theorem.

THEOREM 2.1. The Podles spheres $S_{q \lambda \rho}^{2}$ are slices along the time coordinate of the different regions of the quantum Minkowski space-time $\boldsymbol{M}_{4}$

$$
S_{q \lambda \rho}^{2}=\left\{X_{N} \in \mathcal{M}_{4} \mid X_{0}=t_{0}, \tau^{2}=\tau_{0}^{2}\right\}
$$

The quantum spheres $S_{q c}^{2}$ where $c=c(n)=-1 /\left(q^{n}+q^{-n}\right)^{2}, n=2,3, \ldots, \infty$, correspond to slices at $t_{0}=-q$ and $\tau_{0}^{2}=q^{1 / 2} / \gamma^{2(L)}$, where $n=L+1, L \geq 1$.

The quantum spheres $S_{q c}^{2}$ where $c \in[0, \infty]$ correspond to slices at $t_{0}=-q$ and $\tau_{0}^{2}=-q^{1 / 2} Q^{2} c$ and $S_{q \infty}^{2}$ corresponds to a slice at $t_{0}=0$ and $\tau_{0}^{2}=-q^{1 / 2} Q^{2}$.

Proof. Due to the fact that $X_{0}$ and $\tau^{2}$ commute with the spacial coordinates $X_{z}, X_{\bar{z}}$ and $X_{3}, X_{0}$ and $\tau^{2}$ can be taken to be constants without contradicting the commutation rules (2.13). If we set $X_{z}=Q e_{1}, X_{3}=e_{0}$, $X_{\bar{z}}=Q e_{-1}, \lambda=\left(q-q^{-1}\right) X_{0}=\left(q-q^{-1}\right) t$ and $\rho=q^{-2} t^{2}-q^{-1 / 2} \tau^{2}$ into (2.11) and (2.13), we see that we recover the algebra generators of the quantum sphere $A\left(S_{q}^{2}\right)$ given by $[11,(2 \mathrm{a})-(2 \mathrm{~b})]$.

For $t_{0}=-q$ and $\tau_{0}^{2}=q^{1 / 2} / \gamma^{2(L)} \geq 0, L \geq 1$, we have $\lambda=1-q^{2}$ and $\rho=$ $R^{2}=1-1 / \gamma^{2(L)}=Q^{2} c+1$ giving $c=c(L)=-1 / Q^{2} \gamma^{2(L)}=c(n)=-1 /\left(q^{n}+\right.$ $\left.q^{-n}\right)^{2}$ where $n=L+1$. These constraints correspond to slices of the past time-like region for finite $L$ or a slice of the past light-cone region for $L=\infty$. They fit with the quantum spheres $S_{q c}^{2}$ with $c=c(n) \leq 0$ which are described by states satisfying (2.18), (2.20), and (2.23) for finite $L \geq 1$ and (2.22) and (2.24) for $L=\infty$.

We follow the same procedure presented in [8] to investigate the Hilbert space $\mathscr{\ell}^{s}$ of space of representations of the space-like region of the Minkowski space-time. The elements $|t, n\rangle, n=0,1, \ldots, \infty$, of the basis 
of $\mathfrak{l}^{s}$ satisfy

$$
\begin{aligned}
X_{0}|t, n\rangle= & t|t, n\rangle, \\
X_{3}|t, n\rangle= & \left(q^{2 n}\left(\frac{Q t}{2} \pm \frac{\left(Q^{2} t^{2}-4 \alpha\right)^{1 / 2}}{2}\right)-q^{-1} t\right)|t, n\rangle, \\
X_{z}|t, n\rangle=-q^{-1}\left(q^{(2 n+2)}\left(\frac{Q t}{2} \pm \frac{\left(Q^{2} t^{2}-4 \alpha\right)^{1 / 2}}{2}\right)\right. & \left.\left(Q t-q^{(2 n+2)}\left(\frac{Q t}{2} \pm \frac{\left(Q^{2} t^{2}-4 \alpha\right)^{1 / 2}}{2}\right)\right)-\alpha\right)^{1 / 2}|t, n+1\rangle, \\
& \quad\left(q^{2 n}\left(\frac{Q t}{2} \pm \frac{\left(Q^{2} t^{2}-4 \alpha\right)^{1 / 2}}{2}\right)\right. \\
X_{\bar{z}}|t, n\rangle=-q^{-1} & \left.\quad\left(Q t-q^{2 n}\left(\frac{Q t}{2} \pm \frac{\left(Q^{2} t^{2}-4 \alpha\right)^{1 / 2}}{2}\right)\right)-\alpha\right)^{1 / 2}|t, n-1\rangle,
\end{aligned}
$$

where $\tau^{2}=q^{-3 / 2} \alpha<0$. By substituting $\left.\alpha=-Q^{2} t^{2} c, c \in\right] 0, \infty[$, we get

$$
\begin{aligned}
X_{3}|t, n\rangle= & q^{-1} t\left(q^{(2 n+1)} Q\left(\frac{1}{2} \pm\left(c+\frac{1}{4}\right)^{1 / 2}\right)-1\right)|t, n\rangle, \\
X_{z}|t, n\rangle=-q^{-1} Q t\left(q^{(2 n+2)}\left(\frac{1}{2} \pm\left(c+\frac{1}{4}\right)^{1 / 2}\right)\right. & \left.\left(1-q^{(2 n+2)}\left(\frac{1}{2} \pm\left(c+\frac{1}{4}\right)^{1 / 2}\right)\right)+C\right)^{1 / 2}|t, n+1\rangle, \\
X_{\bar{z}}|t, n\rangle=-q^{-1} Q t\left(q^{2 n}\left(\frac{1}{2} \pm\left(c+\frac{1}{4}\right)^{1 / 2}\right)\right. & \left.\times\left(1-q^{2 n}\left(\frac{1}{2} \pm\left(c+\frac{1}{4}\right)^{1 / 2}\right)\right)+c\right)^{1 / 2}|t, n-1\rangle .
\end{aligned}
$$

If we put in (2.29) $t=t_{0}=-q$, the Hilbert space states $\mathscr{\ell}^{s}$ can be identified to the space of irreducible representations of the Podleś quantum spheres $S_{q c}^{2}$, where $\left.c \in\right] 0, \infty\left[\right.$ and if we put in (2.27) $t_{0}=0$ and $\alpha=-q^{2} Q^{2}$, we obtain the space of representations of the Podleś quantum sphere $S_{q \infty}^{2}$. 
We may also consider, as for the time-like region, that the Hilbert space $\mathscr{\ell}^{s}$ spanned by $|t, n\rangle$ satisfying (2.29) as space of states describing the evolution in the space-like region of the quantum Minkowski spacetime of a free particle moving with an operator velocity of components $V_{z}=X_{x} / t, V_{\bar{z}}=X_{\bar{z}} / t$, and $V_{3}=X_{3} / t$. In this case, we obtain from (2.29) a length of the velocity $|\vec{v}|_{q}^{2}=-\left(G^{i j} / G^{00}\right) V_{i} V_{j}=1+Q^{2} c>1$ greater than the velocity of the light $|\vec{v}|_{q}^{2}=1$ [8].

Note that from (2.29), (2.18), (2.20), and (2.22), we have

$$
\lim _{c \rightarrow 0} \mathscr{H}^{S} \longrightarrow \mathscr{H}^{(\infty)} \longleftarrow \lim _{L \rightarrow \infty} \mathscr{H}^{(L)}
$$

To investigate the transformations of the quantum sphere states under the $\mathrm{SO}_{q}$ (3) quantum group, we have to construct the Hilbert space states $\mathscr{\ell}_{\mathrm{SO}_{q}(3)}$, where the generators $\bar{\Lambda}_{i}{ }^{j}$ act. Since the $X_{0}^{\prime}=\bar{\Delta}_{(L)}\left(X_{0}\right)=I \otimes X_{0}$ and $X_{i}^{\prime}=\bar{\Delta}_{(L)}\left(X_{i}\right)=\bar{\Lambda}_{i}^{j} \otimes X_{j}$ fulfil the same commutation rules (2.13) the transformed states of the quantum sphere satisfy the same relations (2.18) and (2.20) in the time-like region, (2.22) in the light-cone and (2.27) in the space-like region. Since the coordinates $X_{i}$ transform under the tensorial product of $\mathrm{SO}_{q}(3)$ and $S_{q}^{2}$, the transformed states also belong to the tensorial product $\mathscr{H}_{\mathrm{SO}_{q}(3)} \otimes \mathscr{H}_{S_{q}^{2}}$ [8] which needs the construction of the Hilbert space $\mathscr{H}_{\mathrm{SO}_{q}(3)}$.

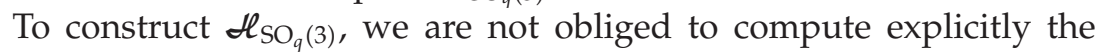
complicated commutation rules (2.8) where we impose (2.14) but we simply consider the action of the $\mathrm{SU}_{q}(2)$ generators on the orthonormal Hilbert space states; $\gamma|n\rangle=q^{n}|n\rangle, \gamma^{\star}|n\rangle=q^{n}|n\rangle, \alpha|n\rangle=\left(1-q^{2 n}\right)^{1 / 2}|n-1\rangle$ and $\alpha^{\star}|n\rangle=\left(1-q^{2(n+1)}\right)^{1 / 2}|n+1\rangle(n=0,1,2, \ldots, \infty)$ which, combined with (2.17), give the action of the generators of $\mathrm{SO}_{q}(3)$ on the basis $|n\rangle$ of the Hilbert space $\mathscr{H}_{\mathrm{SO}_{q}(3)}$ as

$$
\begin{aligned}
& \bar{\Lambda}_{3}^{3}|n\rangle=\left(1-q^{(2 n+1)} Q\right)|n\rangle, \\
& \bar{\Lambda}_{z}^{z}|n\rangle=-2 q^{(2 n+1)}|n\rangle, \\
& \bar{\Lambda}_{\bar{z}} \bar{z}|n\rangle=-2 q^{(2 n+1)}|n\rangle, \\
& \bar{\Lambda}_{3}^{z}|n\rangle=-2 q^{n}\left(1-q^{2 n}\right)^{1 / 2}|n-1\rangle, \\
& \bar{\Lambda}_{3} \bar{z}|n\rangle=-2 q^{(n+1)}\left(1-q^{2(n+1)}\right)^{1 / 2}|n+1\rangle, \\
& \bar{\Lambda}_{z}^{3}|n\rangle=Q q^{(n+1)}\left(1-q^{2(n+1)}\right)^{1 / 2}|n+1\rangle, \\
& \bar{\Lambda}_{\bar{z}}{ }^{3}|n\rangle=Q q^{n}\left(1-q^{2 n}\right)^{1 / 2}|n-1\rangle, \\
& \bar{\Lambda}_{z} \bar{z}^{2}|n\rangle=2\left(1-q^{2(n+1)}\right)^{1 / 2}\left(1-q^{2(n+2)}\right)^{1 / 2}|n+2\rangle, \\
& \bar{\Lambda}_{\bar{z}}{ }^{z}|n\rangle=2\left(1-q^{2 n}\right)^{1 / 2}\left(1-q^{2(n-1)}\right)^{1 / 2}|n-2\rangle .
\end{aligned}
$$


Now, we are ready to investigate the transformations of the quantum sphere states under the $\mathrm{SO}_{q}(3)$ group.

\section{The transformations of the quantum sphere states}

To investigate the transformed quantum sphere states under the $\mathrm{SO}_{q}(3)$ quantum group, we follow the study of the quantum boost transformations in noncommutative special relativity presented in [7]. We recall that in the boost transformation the four coordinates transform by yielding a change of the time operator $X_{0}$, the length of the three spacialvector $\left(q X_{z} X_{\bar{z}}+q^{-1} X_{\bar{z}} X_{z}\right) / Q+X_{3}^{2}$ and the component $X_{3}$ which leads to a change of the quantum number $L$ and $n$ in the transformed states. Under the $\mathrm{SO}_{q}(3)$ transformations, the spacial coordinates transform but the length $|\vec{X}|_{q}$ of the three-vector and the time operator $X_{0}$ remain invariant. Then under the $\mathrm{SO}_{q}(3)$ group the quantum number $L$ remains fixed but $n$ changes and, therefore, the transformed states $|L, p\rangle, p=0,1, \ldots, L$ may be given either in the Hilbert subspace states $\mathscr{H}_{S_{q}^{2}}^{(L)}$ or in the tensorial product $\mathscr{H}_{\mathrm{SO}_{q}(3)} \otimes \mathscr{H}_{S_{q}^{2}}^{(L)}$. More precisely, if we consider the orthonormal basis $|m, L, n\rangle=|m\rangle \otimes|L, n\rangle$ of the Hilbert space $\mathscr{H}_{\mathrm{SO}_{q}(3)} \otimes \mathscr{\ell}_{S_{q}^{2}}^{(L)}$ or $|m, n\rangle=|m\rangle \otimes|n\rangle$ of the Hilbert space $\mathscr{H}_{\mathrm{SO}_{q}(3)} \otimes \mathscr{H}_{S_{q}^{2}}^{(\infty)}$, we have

$$
\begin{gathered}
\left\langle m^{\prime}, L, n^{\prime} \mid m, L, n\right\rangle=\delta_{m^{\prime}, m} \delta_{n^{\prime}, n} \\
\sum_{m=0}^{m=\infty} \sum_{n=0}^{n=L}|m, L, n\rangle\langle m, L, n|=1
\end{gathered}
$$

for finite $L$ and

$$
\begin{gathered}
\left\langle m^{\prime}, n^{\prime} \mid m, n\right\rangle=\delta_{m^{\prime}, m} \delta_{n^{\prime}, n} \\
\sum_{m=0}^{m=\infty} \sum_{n=0}^{n=\infty}|m, n\rangle\langle m, n|=1
\end{gathered}
$$

for $L=\infty$ from which we deduce that under the coaction of $\mathrm{SO}_{q}(3)$ quantum group, the transformed sphere states may be written as

$$
|L, p\rangle=\sum_{m=0}^{m=\infty} \sum_{n=0}^{n=L}|m, L, n\rangle\langle m, L, n \mid L, p\rangle, \quad p=0,1, \ldots, L
$$

for finite $L$ and

$$
|p\rangle=\sum_{m=0}^{m=\infty} \sum_{n=0}^{n=\infty}|m, n\rangle\langle m, n \mid p\rangle, \quad p=0,1, \ldots, \infty
$$


for $L=\infty$. In the space-like region, we have the same relations (3.2) for the Hilbert space $\mathscr{L}_{\mathrm{SO}_{q}(3)} \otimes \mathscr{L}_{S_{q}^{2}}^{s}$ and the same transformed states (3.4) satisfying (2.27) or (2.29).

The transformations (2.15) of the coordinates act on the states (3.3) as

$$
\begin{aligned}
X_{i}^{\prime}|L, p\rangle & =\bar{\Delta}_{(L)}\left(X_{i}\right)|L, p\rangle \\
& =\sum_{m=0}^{m=\infty} \sum_{n=0}^{n=L}\left(\bar{\Lambda}_{i}^{j} \otimes X_{j}\right)|m, L, n\rangle\langle m, L, n \mid L, p\rangle,
\end{aligned}
$$

where $\left(\bar{\Lambda}_{i}^{j} \otimes X_{j}\right)|m, L, n\rangle=\bar{\Lambda}_{i}{ }^{j}|m\rangle \otimes X_{j}|L, n\rangle$. By using (2.18), (2.20), and (2.31), we deduce from (3.5) the following relation:

$$
\begin{aligned}
& X_{3}^{\prime}|L, p\rangle=q^{-1} t\left(\frac{q^{-(L-2 p)}}{\gamma^{(L)}}-1\right)|L, p\rangle \\
& =\frac{q^{-1} t}{Q \gamma^{(L)}} \sum_{m=0}^{m=\infty} \sum_{n=0}^{n=L}\langle m, L, n \mid L, p\rangle \\
& \times\left(\left(1-q^{(2 m+1)} Q\right)\left(q^{-(L-2 n)} Q-q^{(L+1)}-q^{-(L+1)}\right)|m, L, n\rangle\right. \\
& +Q q^{-(L-n-m+1)}\left(1-q^{2 m}\right)^{1 / 2}\left(1-q^{2 n}\right)^{1 / 2} \\
& \times\left(1-q^{2(L-n+1)}\right)^{1 / 2}|m-1, L, n-1\rangle \\
& +Q q^{-(L-n-m-1)}\left(1-q^{2(m+1)}\right)^{1 / 2}\left(1-q^{2(n+1)}\right)^{1 / 2} \\
& \left.\times\left(1-q^{2(L-n)}\right)^{1 / 2}|m+1, L, n+1\rangle\right), \\
& X_{z}^{\prime}|L, p\rangle=-\frac{q^{-1} t}{\gamma^{(L)}} q^{-(L-p)}\left(1-q^{2(p+1)}\right)^{1 / 2}\left(1-q^{2(L-p)}\right)^{1 / 2}|L, p+1\rangle \\
& =\frac{q^{-1} t}{r^{(L)}} \sum_{m=0}^{m=\infty} \sum_{n=0}^{n=L}\langle m, L, n \mid L, p\rangle \\
& \times\left(q^{(m+1)}\left(1-q^{2(m+1)}\right)^{1 / 2}\right. \\
& \times\left(q^{-(L-2 n)} Q-q^{(L+1)}-q^{-(L+1)}\right)|m+1, L, n\rangle \\
& +q^{-(L-n-2 m)}\left(1-q^{2 n}\right)^{1 / 2} \\
& \times\left(1-q^{2(L-n+1)}\right)^{1 / 2}|m, L, n-1\rangle \\
& -q^{-(L-n)}\left(1-q^{2(m+1)}\right)^{1 / 2}\left(1-q^{2(m+2)}\right)^{1 / 2} \\
& \left.\times\left(1-q^{2(n+1)}\right)^{1 / 2}\left(1-q^{2(L-n)}\right)^{1 / 2}|m+2, L, n+1\rangle\right),
\end{aligned}
$$


The quantum sphere

$$
\begin{aligned}
X_{\bar{z}}^{\prime}|L, p\rangle= & -\frac{q^{-1} t}{\gamma^{(L)}} q^{-(L-p+1)}\left(1-q^{2 p}\right)^{1 / 2}\left(1-q^{2(L-p+1)}\right)^{1 / 2}|L, p-1\rangle \\
=\frac{q^{-1} t}{\gamma^{(L)}} & \sum_{m=0}^{m=\infty} \sum_{n=0}^{n=L}\langle m, L, n \mid L, p\rangle \\
& \times\left(q^{m}\left(1-q^{2 m}\right)^{1 / 2}\left(q^{-(L-2 n)} Q-q^{(L+1)}-q^{-(L+1)}\right)|m-1, L, n\rangle\right. \\
& +q^{-(L-n-2 m-1)}\left(1-q^{2(n+1)}\right)^{1 / 2}\left(1-q^{2(L-n)}\right)^{1 / 2}|m, L, n+1\rangle \\
& -q^{-(L-n+1)}\left(1-q^{2(m-1)}\right)^{1 / 2}\left(1-q^{2 m}\right)^{1 / 2}\left(1-q^{2 n}\right)^{1 / 2} \\
& \left.\times\left(1-q^{2(L-n+1)}\right)^{1 / 2}|m-2, L, n-1\rangle\right)
\end{aligned}
$$

By applying $\langle m, L, n|$ from the left, we get, because of linear independence, the following conditions on $\langle m, L, n \mid L, p\rangle$ :

$$
\begin{aligned}
\left(q^{2 p}-\right. & \left.q^{2 n}-q^{2 m}\left(1-q^{2 n}\right)+q^{(2 m+2 n+2)}\left(1-q^{2(L-n)}\right)\right)\langle m, L, n \mid L, p\rangle \\
= & q^{(m+n+1)}\left(1-q^{2(m+1)}\right)^{1 / 2}\left(1-q^{2(n+1)}\right)^{1 / 2} \\
& \times\left(1-q^{2(L-n)}\right)^{1 / 2}\langle m+1, L, n+1 \mid L, p\rangle \\
& +q^{(m+n-1)}\left(1-q^{2 m}\right)^{1 / 2}\left(1-q^{2 n}\right)^{1 / 2} \\
& \times\left(1-q^{2(L-n+1)}\right)^{1 / 2}\langle m-1, L, n-1 \mid L, p\rangle
\end{aligned}
$$

from (3.6),

$$
\begin{aligned}
-q^{-(L-p)} & \left(1-q^{2(p+1)}\right)^{1 / 2}\left(1-q^{2(L-p)}\right)^{1 / 2}\langle m, L, n \mid L, p+1\rangle \\
= & q^{m}\left(1-q^{2 m}\right)^{1 / 2}\left(q^{-(L-2 n)} Q-q^{(L+1)}-q^{-(L+1)}\right)\langle m-1, L, n \mid L, p\rangle \\
& +q^{-(L-n-2 m-1)}\left(1-q^{2(n+1)}\right)^{1 / 2}\left(1-q^{2(L-n)}\right)^{1 / 2}\langle m, L, n+1 \mid L, p\rangle \\
& -q^{-(L-n+1)}\left(1-q^{2(m-1)}\right)^{1 / 2}\left(1-q^{2 m}\right)^{1 / 2}\left(1-q^{2 n}\right)\left(1-q^{2(L-n+1)}\right)^{1 / 2} \\
& \times\langle m-2, L, n-1 \mid L, p\rangle
\end{aligned}
$$


from (3.7), and

$$
\begin{aligned}
-q^{-(L-p+1)}\left(1-q^{2 p}\right)^{1 / 2}\left(1-q^{2(L-p+1)}\right)^{1 / 2}\langle m, L, n \mid L, p-1\rangle \\
=q^{(m+1)}\left(1-q^{2(m+1)}\right)^{1 / 2}\left(q^{-(L-2 n)} Q-q^{(L+1)}-q^{-(L+1)}\right)\langle m+1, L, n \mid L, p\rangle \\
\quad+q^{-(L-n-2 m)}\left(1-q^{2 n}\right)^{1 / 2}\left(1-q^{2(L-n+1)}\right)^{1 / 2}\langle m, L, n-1 \mid L, p\rangle \\
\quad-q^{-(L-n)}\left(1-q^{2(m+1)}\right)^{1 / 2}\left(1-q^{2(m+2)}\right)^{1 / 2}\left(1-q^{2(n+1)}\right)\left(1-q^{2(L-n)}\right)^{1 / 2} \\
\quad \times\langle m+2, L, n+1 \mid L, p\rangle
\end{aligned}
$$

from (3.8). The relations (3.9), (3.10), and (3.11) are the recursion formulas which permit to compute the coefficients $\langle m, L, n \mid L, p\rangle$ giving the transformed states $|L, p\rangle$ in terms of basis elements of the Hilbert space states $\mathscr{H}_{\mathrm{SO}_{q}(3)} \otimes \mathscr{H}_{S_{q}^{2}}^{(L)}$.

To investigate the different coefficients $\langle m, L, n \mid L, p\rangle$, we start by inserting $\langle 0, L, n \mid L, 0\rangle$, and then $\langle m, L, 0 \mid L, 0\rangle$ into (3.9), the result can be iterated $K$ times to get

$$
\begin{aligned}
\langle K, L, n+K \mid L, 0\rangle= & q^{K(n+K)} \prod_{k=1}^{k=K}\left(\frac{\left(1-q^{2(L+1-n-k)}\right)^{1 / 2}}{\left(1-q^{2 k}\right)^{1 / 2}\left(1-q^{2(n+k)}\right)^{1 / 2}}\right) \\
& \times\langle 0, L, n \mid L, 0\rangle, \quad 0 \leq n+K \leq L, \\
\langle m+K, L, K \mid L, 0\rangle= & q^{K(m+K)} \prod_{k=1}^{k=K}\left(\frac{\left(1-q^{2(L+1-k)}\right)^{1 / 2}}{\left(1-q^{2 k}\right)^{1 / 2}\left(1-q^{2(m+k)}\right)^{1 / 2}}\right) \\
& \times\langle m, L, 0 \mid L, 0\rangle, \quad 0 \leq K \leq L .
\end{aligned}
$$

By combining the relations (3.10) and (3.11) with (3.9), we can deduce by a recursive way the coefficients $\langle m+K, L, K \mid L, p+1\rangle,\langle K, L, n+K| L$, $p+1\rangle,\langle m+K, L, K \mid L, p-1\rangle$, and $\langle K, L, n+K \mid L, p-1\rangle$ in terms of those of the development of $|L, p\rangle$ as

$$
\begin{aligned}
\langle m, L, n \mid L, p+1\rangle & \\
= & -q^{(n-p+1)} \frac{\left(1-q^{2(n+1)}\right)^{1 / 2}\left(1-q^{2(L-n)}\right)^{1 / 2}}{\left(1-q^{2(p+1)}\right)^{1 / 2}\left(1-q^{2(L-p)}\right)^{1 / 2}}\langle m, L, n+1 \mid L, p\rangle \\
& -q^{-(m+p-1)} \frac{\left(1-q^{2 m}\right)^{1 / 2}\left(q^{2 p}-q^{n}\right)}{\left(1-q^{2(p+1)}\right)^{1 / 2}\left(1-q^{2(L-p)}\right)^{1 / 2}}\langle m-1, L, n \mid L, p\rangle,
\end{aligned}
$$


The quantum sphere

$$
\begin{aligned}
\langle m, L, n \mid L, p-1\rangle & \\
= & -q^{(n-p+1)} \frac{\left(1-q^{2 n}\right)^{1 / 2}\left(1-q^{2(L-n)}\right)^{1 / 2}}{\left(1-q^{2 p}\right)^{1 / 2}\left(1-q^{2(L+1-p)}\right)^{1 / 2}}\langle m, L, n-1 \mid L, p\rangle \\
& -q^{-(m+p+1)} \frac{\left(1-q^{2(m+1)}\right)^{1 / 2}\left(q^{2 p}-q^{n}\right)}{\left(1-q^{2 p}\right)^{1 / 2}\left(1-q^{2(L+1-p)}\right)^{1 / 2}}\langle m-1, L, n \mid L, p\rangle .
\end{aligned}
$$

Therefore all the coefficients in the development (3.3) of states can be obtained in terms of $\langle m, L, 0 \mid L, 0\rangle$ and $\langle 0, L, n \mid L, 0\rangle$.

Now we are in position to state the following theorem.

THEOREM 3.1. The Hilbert space $\mathscr{H}_{\mathrm{SO}_{q}(3)} \otimes \mathscr{H}_{S_{q}^{2}}^{(L)}$ admits the decomposition

$$
\mathscr{H}_{\mathrm{SO}_{q}(3)} \otimes \mathscr{H}_{S_{q}^{2}}^{(L)}=\sum_{m=-L}^{m=\infty} \oplus \mathscr{H}^{(L, m)},
$$

where the $(L+1)$-dimensional Hilbert subspaces $\mathfrak{L}^{(L, m)}$ are spaces of representations of the same quantum spheres $S_{q c}^{2}$, where $c=c(L)$.

Proof. First, we may see that

$$
p_{(L, m)}=\sum_{K=0}^{K=L}|m+K, L, K\rangle\langle m+K, L, K|, \quad m=-L, \ldots, \infty
$$

are projectors, $p_{(L, m)}^{\dagger}=p_{(L, m)}$ and $p_{(L, m)} p_{\left(L, m^{\prime}\right)}=\delta_{m, m^{\prime}} p_{(L, m)}$, leading to the decomposition

$$
\mathscr{H}_{\mathrm{SO}_{q}(3)} \otimes \mathscr{H}_{S_{q}^{2}}^{(L)}=\sum_{m=-L}^{m=\infty} \oplus \mathscr{H}^{(L, m)} .
$$

For $m<0$ the sum starts from $K=-m$ because for $K+m<0$ the states $|m+K, L, K\rangle$ vanish implying that the dimension of $\mathscr{H}^{(L, m)}$ is $L+1+m$ for $-L \leq m<0$. In this case, we have

$$
\begin{aligned}
|L, p\rangle^{(n)} & =\sum_{K=0}^{L-n}|K, L, n+K\rangle\langle K, L, n+K \mid L, p\rangle \\
& =\sum_{K=n}^{L}|K-n, L, K\rangle\langle K-n, L, K \mid L, p\rangle \in \mathscr{H}^{(L, n)}, \quad n=(1,2, \ldots, L),
\end{aligned}
$$

where $n=-m$. 
Now let in the Hilbert spaces $\mathscr{\ell}^{(L, m)}$ the states

$$
|L, p\rangle^{(m)}=\sum_{K=0}^{K=L}|m+K, L, K\rangle\langle m+K, L, K \mid L, p\rangle .
$$

The normalization condition of these states $|L, 0\rangle^{n}, 0 \leq n \leq L$, and $|L, 0\rangle^{m}$, $m \geq 0$, gives from (3.12)

$$
\begin{aligned}
& (\langle 0, L, n \mid L, 0\rangle)^{2}\left(\sum_{K=0}^{K=L-n} q^{2 K(n+K)} \prod_{k=1}^{k=K}\left(\frac{\left(1-q^{2(L+1-n-k)}\right)^{1 / 2}}{\left(1-q^{2 k}\right)^{1 / 2}\left(1-q^{2(n+k)}\right)^{1 / 2}}\right)\right)=1, \\
& (\langle m, L, 0 \mid L, 0\rangle)^{2}\left(\sum_{K=0}^{K=L} q^{2 K(m+K)} \prod_{k=1}^{k=K}\left(\frac{\left(1-q^{2(L+1-k)}\right)^{1 / 2}}{\left(1-q^{2 k}\right)^{1 / 2}\left(1-q^{2(m+k)}\right)^{1 / 2}}\right)\right)=1,
\end{aligned}
$$

respectively. The restriction of (3.6) to the states (3.18) gives

$$
\begin{aligned}
\bar{\Delta}_{L}\left(X_{3}\right)|L, p\rangle^{(m)} & \\
=\frac{q^{-1} t}{\gamma^{(L)} Q} \sum_{K=0}^{K=L}\langle & +K, L, K|L, p\rangle \\
\times & \left(\left(1-q^{2(m+K)+1} Q\right)\right. \\
& \times\left(q^{-(L-2 K)} Q-q^{(L+1)}-q^{-(L+1)}\right)|m+K, L, K\rangle \\
& +Q q^{-(L-m-2 K+1)}\left(1-q^{2(m+K)}\right)^{1 / 2} \\
& \times\left(1-q^{2 K}\right)^{1 / 2}\left(1-q^{2(L-K+1)}\right)^{1 / 2}|m+K-1, L, K-1\rangle \\
& +Q q^{-(L-m-2 K-1)}\left(1-q^{2(m+K+1)}\right)^{1 / 2}\left(1-q^{2(K+1)}\right)^{1 / 2} \\
& \left.\times\left(1-q^{2(L-K)}\right)^{1 / 2}|m+K+1, L, K+1\rangle\right) .
\end{aligned}
$$

We evaluate this sum by parts. Each part contains a sum constructed by setting $K$ in the first term of the second hand, $K+1$ in the second term and $K-1$ in the third term and gives the same state $|m+K, L, K\rangle$ with 
The quantum sphere

coefficient

$$
\begin{aligned}
\frac{q^{-1} t}{\gamma^{(L)} Q}( & \left(1-q^{2(m+K)+1} Q\right)\left(q^{-(L-2 K)} Q-q^{(L+1)}-q^{-(L+1)}\right)\langle m+K, L, K \mid L, p\rangle \\
& +Q q^{-(L-m-2 K-1)}\left(1-q^{2(m+K+1)}\right)^{1 / 2}\left(1-q^{2(K+1)}\right)^{1 / 2} \\
& \times\left(1-q^{2(L-K)}\right)^{1 / 2}\langle m+K+1, L, K+1 \mid L, p\rangle \\
& +Q q^{-(L-m-2 K+1)}\left(1-q^{2(m+K)}\right)^{1 / 2}\left(1-q^{2 K}\right)^{1 / 2} \\
& \left.\times\left(1-q^{2(L-K+1)}\right)^{1 / 2}\langle m+K-1, L, K-1 \mid L, p\rangle\right) .
\end{aligned}
$$

For $m=m+K$ and $n=K,(3.9)$ reduces to the relation

$$
\begin{aligned}
\left(q^{2 p}-\right. & \left.q^{2 K}-q^{2(m+K)}\left(1-q^{2 K}\right)+q^{(2 m+4 K+2)}\left(1-q^{(L-2 K)}\right)\right)\langle m+K, L, K \mid L, p\rangle \\
= & q^{(m+2 K+1)}\left(1-q^{2(m+K+1)}\right)^{1 / 2}\left(1-q^{2(K+1)}\right)^{1 / 2} \\
& \times\left(1-q^{2(L-K)}\right)^{1 / 2}\langle m+K+1, L, K+1 \mid L, p\rangle \\
& +q^{(m+2 K-1)}\left(1-q^{2(m+K)}\right)^{1 / 2}\left(1-q^{2 K}\right)^{1 / 2} \\
& \times\left(1-q^{2(L-K+1)}\right)^{1 / 2}\langle m+K-1, L, K-1 \mid L, p\rangle
\end{aligned}
$$

which, used into (3.22), leads to the same proportionality coefficient of the state $|m+K, L, K\rangle$ given by

$$
\begin{aligned}
& \frac{q^{-1} t}{\gamma^{(L)} Q}\left(1-q^{2(m+K)+1} Q\right)\left(q^{-(L-2 K)} Q-q^{(L+1)}-q^{-(L+1)}\right) \\
& \quad+Q q^{-L}\left(q^{2 p}-q^{2 K}-q^{2(m+K)}\left(1-q^{2 K}\right)\right. \\
& \left.\quad+q^{(2 m+4 K+2)}\left(1-q^{2(L-K)}\right)\right)\langle m+K, L, K \mid L, p\rangle \\
& =q^{-1} t\left(\frac{q^{-(L-2 p)}}{\gamma^{(L)}}-1\right)\langle m+K, L, K \mid L, p\rangle
\end{aligned}
$$

which leads to

$$
\begin{aligned}
\bar{\Delta}_{L}\left(X_{3}\right)|L, p\rangle^{(m)} & =q^{-1} t\left(\frac{q^{-(L-2 p)}}{\gamma^{(L)}}-1\right) \sum_{K=0}^{K=L}|m+K, L, K\rangle\langle m+K, L, K \mid L, p\rangle \\
& =q^{-1} t\left(\frac{q^{-(L-2 p)}}{\gamma^{(L)}}-1\right)|L, p\rangle^{(m)} .
\end{aligned}
$$


Now if we apply $\bar{\Delta}\left(X_{z}\right)$ on the states (3.18), we obtain

$$
\begin{aligned}
\bar{\Delta}\left(X_{z}\right)|L, p\rangle^{(m)} & \\
=\frac{q^{-1} t}{\gamma^{(L)}} \sum_{K=0}^{K=L}\langle & \\
\times & \left(q^{(m+K+1)}\left(1-q^{2(m+K+1)}\right)^{1 / 2}\right. \\
& \times\left(q^{-(L-2 K)} Q-q^{(L+1)}-q^{-(L+1)}\right)|m+K+1, L, K\rangle \\
& +q^{-(L-2 m-3 K)}\left(1-q^{2 K}\right)^{1 / 2}\left(1-q^{2(L-K+1)}\right)^{1 / 2}|m+K, L, K-1\rangle \\
& -q^{-(L-K)}\left(1-q^{2(m+K+1)}\right)^{1 / 2}\left(1-q^{2(m+K+2)}\right)^{1 / 2} \\
& \left.\times\left(1-q^{2(K+1)}\right)^{1 / 2}\left(1-q^{2(L-K)}\right)^{1 / 2}|m+K+2, L, K+1\rangle\right) .
\end{aligned}
$$

The computation of each part of this sum corresponding to $K$ for the first term of the right-hand side of (3.26), $K+1$ for the second term and $K-1$ for the third term leads to a proportionality coefficient of a same state $|m+1+K, L, K\rangle$ given by

$$
\begin{aligned}
& \frac{q^{-1} t}{\gamma^{(L)}} q^{(m+K+1)}\left(1-q^{2(m+K+1)}\right)^{1 / 2}\left(q^{-(L-2 K)} Q-q^{(L+1)}-q^{-(L+1)}\right) \\
& \quad \times\langle m+1+K-1, L, K \mid L, p\rangle+q^{-(L-2 m-3 K-3)}\left(1-q^{2(K+1)}\right)^{1 / 2} \\
& \quad \times\left(1-q^{2(L-K)}\right)^{1 / 2}\langle m+1+K, L, K+1 \mid L, p\rangle \\
& -q^{-(L-K+1)}\left(1-q^{2(m+K)}\right)^{1 / 2}\left(1-q^{2(m+K+1)}\right)^{1 / 2}\left(1-q^{2 K}\right)^{1 / 2} \\
& \quad \times\left(1-q^{2(L+1-K)}\right)^{1 / 2}\langle m+1+K-2, L, K-1 \mid L, p\rangle .
\end{aligned}
$$

By replacing into (3.10) $m$ by $m+1+K$ and $n$ by $K$, we see that (3.27) reduces to

$$
-\frac{q^{-1} t}{\gamma^{(L)}} q^{-(L-p)}\left(1-q^{2(p+1)}\right)^{1 / 2}\left(1-q^{2(L-p)}\right)^{1 / 2}\langle m+1+K, L, K \mid L, p\rangle
$$


332 The quantum sphere

which shows that (3.26) reads

$$
\begin{aligned}
\bar{\Delta}_{L}\left(X_{z}\right)|L, p\rangle^{(m)}= & -\frac{q^{-1} t}{\gamma^{(L)}} q^{-(L-p)}\left(1-q^{2(p+1)}\right)^{1 / 2}\left(1-q^{2(L-p)}\right)^{1 / 2} \\
& \times \sum_{K=0}^{K=L}|m+1+K, L, K\rangle\langle m+1+K, L, K \mid L, p+1\rangle \\
= & -\frac{q^{-1} t}{\gamma^{(L)}} q^{-(L-p)}\left(1-q^{2(p+1)}\right)^{1 / 2}\left(1-q^{2(L-p)}\right)^{1 / 2}|L, p+1\rangle^{(m+1)} .
\end{aligned}
$$

The same way gives

$$
\begin{aligned}
\bar{\Delta}_{L}\left(X_{\bar{z}}\right)|L, p\rangle^{(m)}= & -\frac{q^{-1} t}{\gamma^{(L)}} q^{-(L-p+1)}\left(1-q^{2 p}\right)^{1 / 2}\left(1-q^{2(L-p+1)}\right)^{1 / 2} \\
& \times \sum_{K=0}^{K=L}|m-1+K, L, K\rangle\langle m-1+K, L, K \mid L, p-1\rangle \\
= & -\frac{q^{-1} t}{\gamma^{(L)}} q^{-(L-p+1)}\left(1-q^{2 p}\right)^{1 / 2}\left(1-q^{2(L-p+1)}\right)^{1 / 2}|L, p-1\rangle^{(m-1)}
\end{aligned}
$$

By using (3.25), (3.29), and (3.30), we can show from a straightforward computation that the states $|L, p\rangle^{(m)}, m=-L,-L+1, \ldots, \infty$, are eigenstates of $\left(q X_{z}+q^{-1} X_{\bar{z}}\right) / Q+X_{3}^{2}$ with the same eigenvalue $q^{-2} t^{2}\left(1-1 / \gamma^{2(L)}\right)=$ $R^{2(L)}$.

Note that for $m=0$ and $n=L$ (3.9) gives $\left(q^{2 p}-1\right)\langle 0, L, L \mid L, p\rangle=0$ yielding

$$
\langle 0, L, L \mid L, p\rangle=0 \quad \text { if } p>0 \text {. }
$$

On the other hand, (3.19) leads to

$$
(\langle 0, L, L \mid L, 0\rangle)^{2}=1
$$

yielding $\langle 0, L, L \mid L, 0\rangle=\lambda$ with $\lambda \bar{\lambda}=1$. If we take $\lambda=1$, we get

$$
|L, 0\rangle(L)=|0, L, L\rangle\langle 0, L, L \mid L, 0\rangle=|0, L, L\rangle
$$


which is the unique state of the one-dimensional Hilbert subspace $\mathscr{\ell}^{(L,-L)}$. On the other hand, for $m=0,(3.13)$ gives

$$
\langle 0, L, n \mid L, p+1\rangle=-q^{(n-p+1)} \frac{\left(1-q^{2(n+1)}\right)^{1 / 2}\left(1-q^{2(L-n)}\right)^{1 / 2}}{\left(1-q^{2(p+1)}\right)^{1 / 2}\left(1-q^{2(L-p)}\right)^{1 / 2}}\langle 0, L, n+1 \mid L, p\rangle
$$

which shows that $\langle 0, L, L-1 \mid L, p+1\rangle=0$ if $p>0$ or $\langle 0, L, L-1 \mid L, p\rangle=0$ if $p>1$. By iteration, we get from (3.34)

$$
\langle 0, L, L-k \mid L, p\rangle=0 \quad \text { if } p>k
$$

Now by substituting (3.35) into the left-hand side of (3.9), we get

$$
\langle K, L, L-k+K \mid L, p\rangle=0 \quad \text { if } p>k
$$

and by setting $L-k=n$, we obtain

$$
\langle K, L, n+K \mid L, p\rangle=0 \Longrightarrow|L, p\rangle^{(n)}=0 \quad \text { for } L-n<p \leq L
$$

Then the Hilbert subspace states $\mathscr{\ell}^{(L, m)},-L \leq m \leq 1$, do not describe the whole of the quantum sphere but only its parts described by the states $|L, 0\rangle, \ldots,|L, L+m\rangle$. Equations (3.29) and (3.30) show that $X_{z}$ is a linear mapping $X_{z}: \mathscr{\ell}^{(L, m)} \rightarrow \mathscr{\ell}^{(L, m+1)}$ and $X_{\bar{z}}$ is a linear mapping $X_{\bar{z}}$ : $\mathscr{H}^{(L, m)} \rightarrow \mathscr{H}^{(L, m-1)}$. To obtain the Hilbert space of representations, we consider the orthogonal subspaces $\mathscr{H}^{(L, m)} m=-L, \ldots, \infty$ spanned by the bases $|L, p\rangle^{(m+p)}$ with $p=0,1, \ldots, L$ which are the Hilbert subspaces in the decomposition (3.14). The $(L+1)$-dimensional subspaces $\mathscr{L}^{(L, m)}$ are irreducible space representations of the same quantum sphere $S_{q c}^{2}$, where $c=c(L)$.

Note that by using the eigenstate relations (2.22) and the relations (2.31), the same procedure shows that in the light-cone $(L=\infty)$, the states

$$
|p\rangle^{(m)}=\sum_{K=0}^{K=\infty}|m+K, K\rangle\langle m+K, K \mid p\rangle, \quad m+K \geq 0
$$


satisfy

$$
\begin{aligned}
\bar{\Delta}_{L}\left(X_{3}\right)|p\rangle^{(m)}= & q^{-1} t\left(q^{(2 p+1)} Q-1\right) \sum_{K=0}^{K=\infty}|m+K, K\rangle\langle m+K, K \mid p\rangle \\
= & q^{-1} t\left(q^{(2 p+1)} Q-1\right)|p\rangle^{(m)}, \\
\bar{\Delta}_{L}\left(X_{z}\right)|p\rangle^{(m)}= & -q^{-1} t Q q^{(p+1)}\left(1-q^{2(p+1)}\right)^{1 / 2} \sum_{K=0}^{K=\infty}|m+1+K, K\rangle \\
& \times\langle m+1+K, K \mid p+1\rangle \\
= & -q^{-1} t Q q^{(p+1)}\left(1-q^{2(p+1)}\right)^{1 / 2}|p+1\rangle^{(m+1)}, \\
\bar{\Delta}_{L}\left(X_{\bar{z}}\right)|p\rangle^{(m)}= & -q^{-1} t Q q^{p}\left(1-q^{2 p}\right)^{1 / 2} \sum_{K=0}^{K=\infty}|m-1+K, K\rangle \\
& \times\langle m-1+K, K \mid p-1\rangle \\
= & -q^{-1} t Q q^{p}\left(1-q^{2 p}\right)^{1 / 2}|p-1\rangle^{(m-1)} .
\end{aligned}
$$

The states $|p\rangle^{(m)}$ are eigenstates of $\left(q X_{z}+q^{-1} X_{\bar{z}}\right) / Q+X_{3}^{2}$ with the same eigenvalue $q^{-2} t^{2}$. In this case, we consider, in the decomposition (3.14) the subspaces $\mathscr{L}^{(m)}$ spanned by the bases $|p\rangle^{(m+p)} p=0,1, \ldots, \infty$. The same procedure may be done in the space-like region for the Hilbert space $\mathscr{H}_{S_{q}^{2}}^{s}$.

\section{Conclusion}

In this paper, we showed that the different Podleś spheres can be obtained as slices along the time coordinate of the different regions (light-cone, time-like or space-like) of the quantum Minkowski spacetime. The representation of the coaction of the $\mathrm{SO}_{q}(3)$ quantum group on the quantum spheres $S_{q c}^{2}$ in the Hilbert space states exhibits the periodicity of the quantum sphere states through a decomposition (3.14) of the Hilbert space transformed states in terms of orthogonal Hilbert subspaces, each of them being a space of states of a same quantum sphere. The state transformations in time-like region (finite $L$ ) or light-cone $(L=\infty)$ may also be regarded as transformations under the $\mathrm{SO}_{q}(3)$ quantum subgroup of the Lorentz group of states describing the evolution in the quantum Minkowski space-time of a free particle moving with a velocity of the length $|\vec{v}|_{q}=\left(1-1 / \gamma^{2(L)}\right)^{1 / 2}$ and of component $v_{3}^{(L, n)}=q^{-1}\left(q^{-(L-2 n)} / \gamma^{(L)}-1\right)$ for finite $L$ and of the length $|\vec{v}|_{q}=1$, velocity of the light, and of component $v_{3}^{(n)}=q^{-1}\left(q^{(2 n+1)} Q-1\right)$ for $L=\infty$. 


\section{Acknowledgment}

I am particularly grateful to the Abdus Salam International Centre for Theoretical Physics, Trieste, Italy, for hospitality.

\section{References}

[1] T. Brzeziński and S. Majid, Quantum group gauge theory on quantum spaces, Comm. Math. Phys. 157 (1993), no. 3, 591-638, Erratum: Comm. Math. Phys. 167 (1995), no. 1, 235.

[2] C.-S. Chu, P.-M. Ho, and B. Zumino, Some complex quantum manifolds and their geometry, Quantum Fields and Quantum Space Time (Cargèse, 1996), NATO Advanced Science Institutes Series B: Physics, vol. 364, Plenum, New York, 1997, pp. 281-322.

[3] H. Grosse, J. Madore, and H. Steinacker, Field theory on the q-deformed fuzzy sphere II: Quantization, preprint, 2001, http://arxiv.org/abs/ hep-th/0103164.

[4] , Field theory on the q-deformed fuzzy sphere. I, J. Geom. Phys. 38 (2001), no. 3-4, 308-342.

[5] P. M. Hajac, Strong connections on quantum principal bundles, Comm. Math. Phys. 182 (1996), no. 3, 579-617.

[6] P. M. Hajac and S. Majid, Projective module description of the q-monopole, Comm. Math. Phys. 206 (1999), no. 2, 247-264.

[7] M. Lagraa, The boosts in the noncommutative special relativity, preprint, 2001, http:/ /arxiv.org/abs/math-ph/0103033.

[8] - Measurability of observables in the noncommutative special relativity, preprint, 2001, http:/ /arxiv.org/abs/math-ph/9904014.

[9] _ On the quantum Lorentz group, J. Geom. Phys. 34 (2000), no. 3-4, 206225.

[10] A. Pinzul and A. Stern, Dirac operator on the quantum sphere, Phys. Lett. B 512 (2001), no. 1-2, 217-224.

[11] P. Podleś, Quantum spheres, Lett. Math. Phys. 14 (1987), no. 3, 193-202.

[12] - Symmetries of quantum spaces. Subgroups and quotient spaces of quantum SU(2) and SO(3) groups, Comm. Math. Phys. 170 (1995), no. 1, 1-20.

M. Lagraa: Laboratoire de Physique Théorique, Université d'Oran Es-Sénia, 31100, Algérie

Current address: Abdus Salam International Centre for Theoretical Physics, Trieste, Italy 


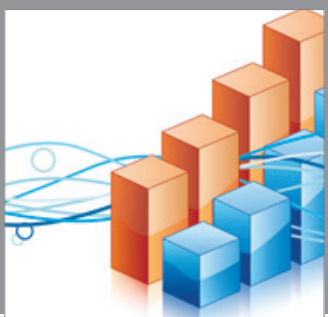

Advances in

Operations Research

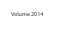

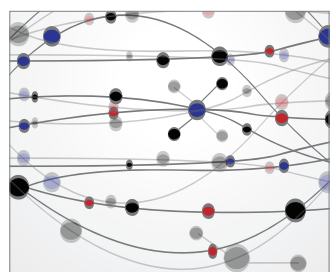

\section{The Scientific} World Journal
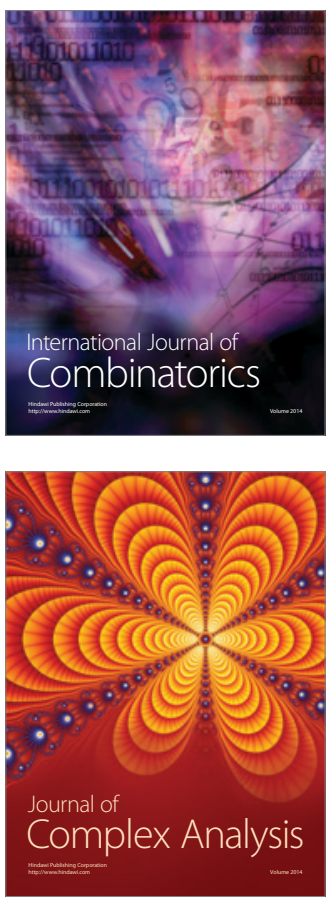

International Journal of

Mathematics and

Mathematical

Sciences
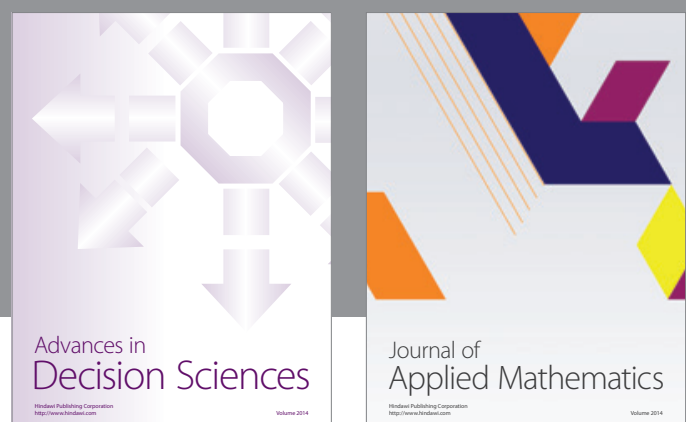

Journal of

Applied Mathematics
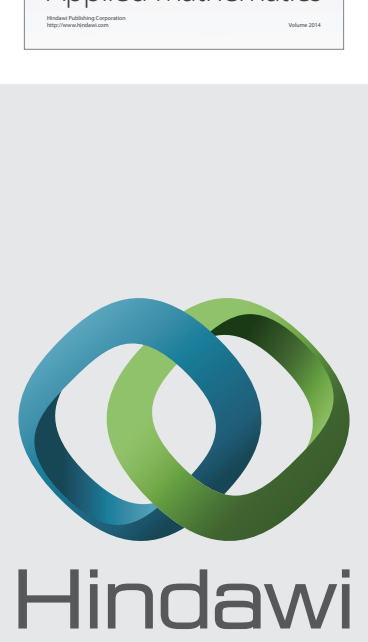

Submit your manuscripts at http://www.hindawi.com
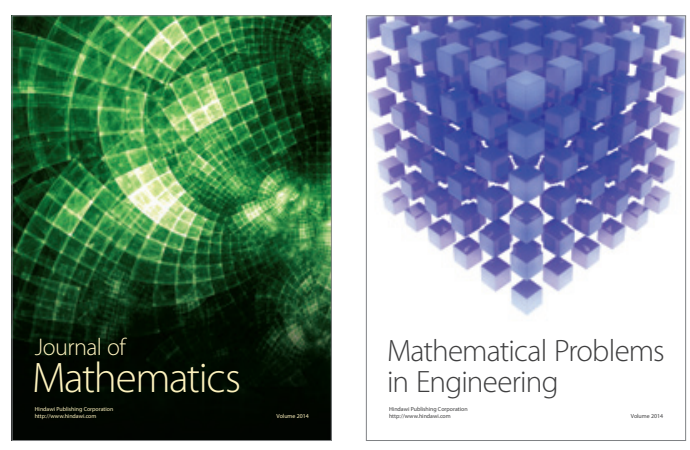

Mathematical Problems in Engineering
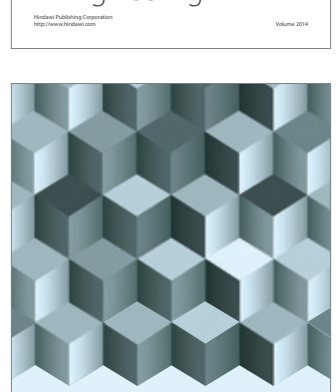

Journal of

Function Spaces
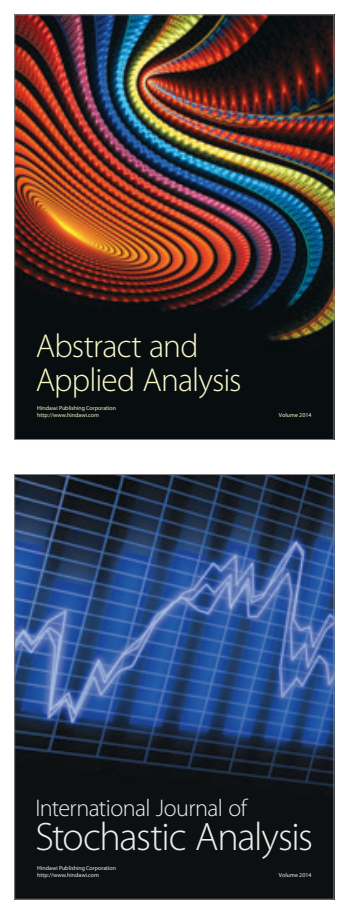

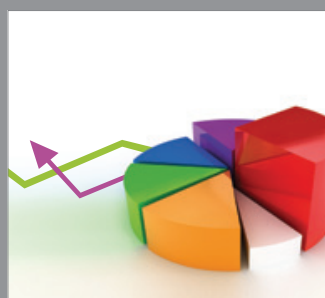

ournal of

Probability and Statistics

Promensencen
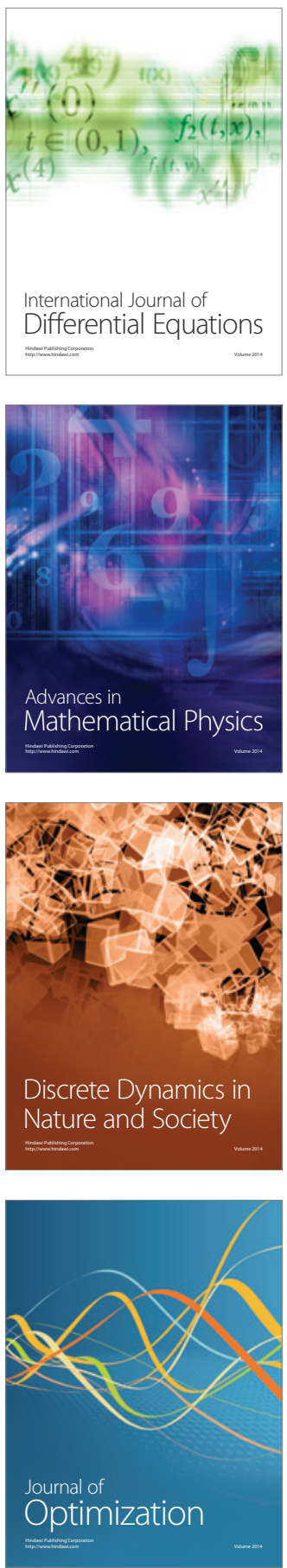